Rev. Saúde públ., S. Paulo, 24(5):380-6, 1990

\title{
PREVALÊNCIA DE ANEMIA EM GESTANTES DE PRIMEIRA CONSULTA EM CENTROS DE SAÚDE DE ÁREA METROPOLITANA, BRASIL*
}

\author{
Elvira Maria Guerra** \\ Orlando César de Oliveira Barretto*** \\ Adelaide José Vaz**** \\ Maria Beatriz Silveira**
}

GUERRA, E. M. et al. Prevalência de anemia em gestantes de primeira consulta em centros de saúde de área metropolitana, Brasil. Rev. Saúde públ., S. Paulo, 24: 380-6, 1990.

RESUMO: No periodo de abril a outubro de 1988, foram estudadas 363 gestantes de primeira consulta, as quais estavam inscritas no Programa de Atendimento à Gestante da Secretaria do Estado da Saúde de Såo Paulo, subdistrito do Butantă, Município de Săo Paulo, Brasil. Na ocasiăo da coleta do material biológico estas gestantes năo faziam uso de medicamentos contendo ferro, ácido fólico, vitamina B12 ou associaçóes destes. A idade média das gestantes foi de 25 anos, 65,9\% delas pertenciam a familias com renda até 1 SMPC (salário mínimo per capita) e apenas 3,1\% pertenciam a familias com renda superior a 3 SMPC. A prevalência de anemia foi de $12,4 \%$. A média da concentração de hemoglobina $(\mathrm{g} / \mathrm{dl})$ no primeiro trimestre foi significativamente maior que as médias no segundo e terceiro trimestres gestacional. A prevalência de anemia no primeiro trimestre: $(3,6 \%)$ foi significativamente menor do que aquelas encontradas no segundo $(20,9 \%)$ e terceiro trimestre $(32,1 \%)$. As gestantes que tiveram mais que três partos tiveram prevalência de anemia significativamente maior do que aquelas com até três partos. $\mathrm{A}$ prevalência de anemia foi maior no grupo das gestantes que pertenciam a familias com renda até 0,5 SMPC. análise.

DESCRITORES: Anemia hipocrômica, epidemiologia. Complicações na gravidez. Hemoglobinas,

\section{INTRODUÇÃO}

A anemia é definida como a redução da capacidade de transporte de oxigênio do sangue, devido à diminuição do número de eritrócitos, do conteúdo hemoglobínico ou de ambos.

A Organização Mundial da Saúde verificou que 21 a $80 \%$ das gestantes, em diferentes partes do mundo, săo anểmicas ${ }^{38}$ e se refere à anemia severa e à desnutrição como fatores que afetam o desenvolvimento da gravidez, afetando o crescimento fetal e o peso ao nascer, contribuindo significativamente na mortalidade perinatal ${ }^{39}$.

Royston 26 verificou em sua revisão que por ordem de freqüência, a prevalência de anemia é maior na Ásia, seguida pela África e América Latina.

Demayer e Adiels-Tegman ${ }^{8}$ fizeram revisão sobre a prevalência de anemia no mundo, e puderam estimar que a prevalência total é de $30 \%$, sendo as crianças e as gestantes os grupos mais afetados. Esses autores observaram que a prevalência de anemia em gestantes de regiбes em desenvolvimento é por volta de $54 \%$ e nas regióes desenvolvidas (América do Norte, Japão, Europa, Austrália, Nova Zelândia e URSS) é de $9 \%$.

A prevalência de anemia em gestantes no Brasil tem sido estudada por vários autores 5, 20, 24, 25, 27, $32,33,34,36,37$ e os resultados encontrados têm variado de acordo com: critérios de seleção da amostragem; idade gestacional; condições sócio-econômicas das gestantes; estado de saneamento básico da região estudada.

A Secretaria da Saúde do Estado de São Paulo mantém o Programa de Atendimento à Gestante nos Centros de Saúde, no qual são solicitados, na inscrição ou na primeira consulta médica, exames laboratoriais de rotina ${ }^{29}$.

\footnotetext{
* Síntese da dissertação de Mestrado: "Prevalência de anemia em gestantes de ṕrimeira consulta em Centros de Saúde do Estado no Subdistrito de Paz do Butantā, Município de São Paulo", na Faculdade de Ciências Farmacêuticas da Universidade de São Paulo.

* Instituto Adolfo Lutz. Divisão de Patologia - Seção de Hematologia - Av. Arnaldo, 351 - 01246 - São Paulo, SP Brasil.

** Faculdade de Medicina da Universidade de São Paulo. Laboratório de Pesquisas Hematológicas - Av. Dr. Arnaldo, 455 - 01246 - São Paulo, SP - Brasil.

**** Instituto Adolfo Lutz. Divisão de Biologia Médica - Laboratório de Imunodiagnóstico. São Paulo, SP - Brasil.
} 
O objetivo do presente trabalho foi de estudar a prevalência de anemia em gestantes de primeira consulta na rede pública, no subdistrito do Butantan, na tentativa de contribuir para eventuais modificaçōes nas açőes da Secretaria da Saúde para prevençăo da anemia neste grupo.

\section{CASUISTICA E MÉTODOS}

Do total de 481 gestantes consecutivas, de primeira consulta, inscritas no Programa de Atendimento à Gestante da Secretaria da Saúde de São Paulo, 118 não foram incluídas no presente estudo, pois na ocasião da coleta do material biologico as gestantes faziam uso de medicamentos que continham ferro, ácido fólico, vitamina B12 ou associações destes. O número da amostra considerado representativo foi de 360 e o total das gestantes estudadas foi de 363 .

As gestantes estavam matriculadas em oito Centros de Saúde do Estado, no subdistrito do Butantan, localizado na zona oeste, área periférica, do Município de São Paulo. Nesse subdistrito está situado o Campus da Cidade Universitária da Universidade de São Paulo, contrastando com a presença de grande número de favelas ${ }^{10} \mathrm{e}$ infraestrutura básica carente ${ }^{28}$.

Para dosagem de hemoglobina foram colhidas amostras de $3 \mathrm{ml}$ de sangue de cada gestante, em tubo contendo anticoagulante etileno-diaminotetracético de potássio (Sistema Vacutainer).

A dosagem de hemoglobina foi realizada pelo método de cianometahemoglobina ${ }^{7}$ e foram realizadas em duplicata por uma só pessoa.

Foi aplicado um questionário à populaçăo estudada, contendo as informaçōes: idade da gestante, grupo étnico, Estado de origem, idade gestacional, paridade, intervalo do último parto, uso de medicamentos e tempo de uso, renda familiar e número de pessoas que compunha a família da gestante.

Como a cidade de São Paulo está situada a 835 metros do nível do mar, considerou-se existir anemia quando a concentração de hemoglobina ( $\mathrm{g} / \mathrm{dl}$ ) foi inferior a $11,6 \mathrm{~g} / \mathrm{dl}$, pois somou-se $0,2 \mathrm{~g} / \mathrm{dl}$ para cada $300 \mathrm{~m}$ de altitude ${ }^{11}$ aquela hemoglobina considerada normal ao nível do mar de $11,0 \mathrm{~g} / \mathrm{dl}^{38}$.

$\mathrm{Na}$ variável renda utilizamos o salário mínimo per capita (SMPC), o qual foi obtido através de duas divisões. Primeiro dividimos a renda familiar dada em cruzados pelo piso nacional de salários do mês da aplicação do questionário. Após, a renda familiar em salários mínimos foi dividida pelo número de pessoas que compunha a familia da gestante, inclusive as crianças, obtendo assim o SMPC. O valor do piso nacional de salários em cruzados foi dividido pelo valor ofi- cial do dólar americano no dia 30 de cada mês, obtendo-se o valor do piso nacional do salário, em dólares americanos (média).

$\mathrm{Na}$ análise dos dados, as freqüências foram obtidas através do programa de banco de dados DBASE III PLUS para microcomputador IBM PC XT compatível. A análise estatística foi realizada através dos seguintes métodos:

- análise de variância para médias independentes, quando era de nosso interesse estudar as variáveis idade das gestantes e concentração de hemoglobina segundo o trimestre gestacional ${ }^{3}$;

- teste T-Student para analisar as médias da concentração de hemoglobina ( $\mathrm{g} / \mathrm{dl}$ ) no grupo das gestantes primigestas e multigestas, segundo o trimestre gestacional ${ }^{3}$;

- teste do Qui-quadrado para comparar as prevalências de anemia frente as diferentes variáveis: trimestre de gestação, número de partos e renda dada em salário mínimo per capita 30 .

Em todos os testes estatísticos o nível de significância foi sempre igual ou menor que $0,05(p<0,05)$.

\section{RESULTADOS E COMENTÁRIOS}

A idade média das gestantes foi de 25 anos, com idade mínima de 14 e máxima de 46 anos. Não houve diferença significativa entre as médias das idades das gestantes nos três trimestres de gestação, mostrando ser a amostra homogênea quanto a este aspecto (Tabela 1).

\section{TABELA 1}

Caracterização do grupo total quanto as médias das idades das gestantes, médias da concentraçăo da hemoglobina $(\mathrm{g} / \mathrm{dl})$, prevalências de anemia, segundo o trimestre gestacional.

\begin{tabular}{lccc}
\hline & \multicolumn{3}{c}{ Idade Gestacional (Trimestre) } \\
\cline { 2 - 4 } $\begin{array}{c}\text { Caracteristicas } \\
\text { das gestantes }\end{array}$ & I & II & III \\
\hline número de gestantes & 196 & 139 & 28 \\
idade* $_{\text {média Hb (g/dl) }}$ & 24,27 & 25,01 & 23,75 \\
prevalência de & $13,47^{* *}$ & 12,47 & 12,25 \\
anemia(\%) & $3,6^{* * *}$ & 20,9 & 32,1 \\
\hline
\end{tabular}

Análise de variância entre as médias das idades das gestantes: não significativa.

* Análise de variância entre as médias das concentrações de hemoglobina: a média de concentração de hemoglobina do primeiro trimestre é significativamente maior que a do segundo e terceiro trimestres $(p<0,05)$.

** Teste do Qui-quadrado entre as prevalências de anemia: a prevalência de anemia no primeiro trimestre é significativamente menor do que as do segundo e terceiro trimestres $(p<0,05)$. 
Das 363 gestantes, $188(51,8 \%)$ eram caucasóides e $175(48,2 \%)$ negróides. Não encontramos nenhuma gestante mongolóide no grupo estudado, provavelmente, devido às características sócioeconômicas deste grupo. Apenas $33,6 \%$ das gestantes eram nascidas no Estado de São Paulo. Do restante, $11,6 \%$ eram procedentes de outros Estados da região sudeste, $44,6 \%$ da região nordeste, $8,8 \%$ da região sul, $1,1 \%$ da região centro-oeste do Brasil e $0,3 \%$ era proveniente do Chile, caracterizando uma população de migrantes de outros Estados.

Das gestantes que declararam renda familiar $(\mathrm{n}=358), 65,9 \%$ pertenciam a famílias com renda até um SMPC, $31,0 \%$ a famílias com renda maior que 1,0 até $3,0 \mathrm{SMPC}$ e apenas $3,1 \%$ pertenciam a famílias com renda superior a 3,0 SMPC. $O$ salário mínimo per capita médio foi de 0,98 . No período de abril a outubro de 1988 , um piso nacional de salário correspondia, em média, a US\$ 52,3, tendo variado de US\$ 50,1 a US\$ 53,5.

Encontramos que $54 \%$ das gestantes procuraram - Serviço de Pré-Natal logo no início da gestação, isto é, no primeiro trimestre gestacional; $38,3 \%$ estavam no segundo trimestre e somente $7,7 \%$ das gestantes procuraram a rede para atendimento de pré-natal no terceiro trimestre gestacional. Este fato difere da população estudada por Vaz Pinto e col. ${ }^{37}$, na cidade satélite de Sobradinho (DF), na qual predominou gestantes de terceiro trimestre de gestação. Esta discrepência possivelmente se deve a maior oferta de serviços de saúde gratuitos na região por nós estudada, o que poderia explicar a maior precocidade na procura de atendimento médico por parte da gestante.

A prevalência de anemia em gestantes de primeira consulta na rede estadual, no subdistrito do Butantan, Município de São Paulo, foi de $12,4 \%$. Esta prevalência é semelhante àquelas encontradas por Szarfarc e col..$^{34}$, para o grupo de gestantes com atendimento de pré-natal em diferentes serviços de saúde em São Paulo; Romani e col. ${ }^{24}$, para o grupo de gestantes do PAM-Areias na cidade de Recife; Szarfarc ${ }^{36}$, para o grupo de gestantes pertencentes ao Centro de Saúde da Lapa (Município de São Paulo); Lira e col. ${ }^{17}$, em gestantes do Distrito de Santiago no Chile; Grebe e col..$^{13} \mathrm{em}$ gestantes da região sul-oriente de Santiago do Chile; Flores e col..$^{12}$ em gestantes de Três Rios, na Costa Rica; Coulibaly e col. ${ }^{6}$ em gestantes no Norte de Cameroon na África; e Ho e col. ${ }^{14}$ em gestantes na China. $\mathrm{E}$ é menor do que aquelas encontradas por Cook e col..$^{5}$ em gestantes da América Latina; Szarfarc ${ }^{32}$ em parturientes da Casa Maternal e de Assistência à Infância da Legião Brasileira de Assistência na cidade de São Paulo; Vaz Pinto e col. ${ }^{37}$ em gestantes da cidade satélite de Sobradinho (DF); Salzano e col. ${ }^{27} \mathrm{em}$ gestantes dos Estados de Pernambuco e Paraíba; Szarfarc e col..$^{34}$ em gestantes sem atendimento de pré-natal na cidade de São Paulo; Romani e col. ${ }^{24} \mathrm{em}$ gestantes inscritas no Cen- tro de Saúde Lessa de Andrade na cidade de Recife; Szarfarc $^{36}$ (1985) em gestantes inscritas no Programa de Atendimento à Gestante dos Centros de Saúde da Secretaria do Estado da Saúde no Estado de São Paulo; Diez-Ewald e Molina ${ }^{9}$ em gestantes em Maracaibo na Venezuela; Lira e col. ${ }^{18} \mathrm{em}$ Santiago no Chile; Cardozo e col. ${ }^{4}$ em Santa Cruz, Bolívia; Shukla e col. ${ }^{31} \mathrm{em}$ Utta Pradesh, India.

E a prevalência de anemia por nós encontrada é maior que aquela encontrada por Szarfarc e col. ${ }^{34}$ no grupo de gestantes que realizavam o pré-natal re-gularmente no Centro de Saúde Escola Geraldo Paula Souza, no Município de São Paulo.

A caracterização do grupo total quanto a média das idades das gestantes, idade gestacional, médias da concentração de hemoglobina $(\mathrm{g} / \mathrm{dl})$ e prevalência de anemia segundo o trimestre gestacional são apresentados na Tabela 1.

A prevalência de anemia segundo o trimestre de gestação, na amostra estudada, foi de $3,6 \%$ das gestantes de primeiro trimestre, $20,9 \%$ das de segundo trimestre e $32,1 \%$, de terceiro trimestre.

Vaz Pinto e col..$^{37}$ encontraram as seguintes prevalências de anemia nas gestantes da cidade satélite de Sobradinho: $22 \%$ no primeiro trimestre, $29 \%$ no segundo e $34 \%$ no terceiro.

Diez-Ewald e Molina9 observaram anemia em $20 \%$ das gestantes de primeiro trimestre, em $34 \%$ das de segundo trimestre e $53 \%$ de terceiro trimestre.

Flores e col..$^{12}$ encontraram as seguintes prevalências de anemia: $12,5 \%$ no primeiro, $10,0 \%$ no segundo e $24 \%$ no terceiro trimestre de gestação.

As gestantes de primeiro trimestre apresentaram a média da concentração de hemoglobina $(13,47 \mathrm{~g} / \mathrm{dl})$ significativamente maior que as médias do segundo $(12,47 \mathrm{~g} / \mathrm{dl})$ e terceiro $(12,25 \mathrm{~g} / \mathrm{dl})$ trimestres. Estas médias são maiores que aquelas encontradas por Almeida e col. ${ }^{1}$, Szarfarc ${ }^{36}$, Isah e col. $^{15}$, Mayet ${ }^{21}$, Razvi e col. ${ }^{23}$, Flores e col. ${ }^{12}$, Shukla e col $^{31} \mathrm{e}$ semelhantes às médias encontradas por Vaz Pinto e col. ${ }^{37}$, Szarfarc e col. ${ }^{35}$, Loria e col. ${ }^{19} \mathrm{e}$ Lamparelli e col. ${ }^{16}$.

Em relação a paridade, $151(41,6 \%)$ gestantes eram primigestas e $212(58,4 \%)$ multigestas. $\mathrm{Na}$ Tabela 2 são apresentadas as médias da concentração de hemoglobina $(\mathrm{g} / \mathrm{dl})$, as prevalências de anemia segundo o trimestre gestacional das gestantes primigestas e multigestas. Não houve diferença significativa entre as médias das concentraçôes de hemoglobina das gestantes primigestas e multigestas, em cada trimestre de gestação.

As gestantes primigestas e as multigestas apresentaram prevalência de anemia de $9,93 \%$ e $14,15 \%$, respectivamente, não havendo diferença significativa entre os grupos. 
TABELA 2

Caracterização das gestantes primigestas e multigestas quanto ao número de gestantes, médias das concentraçoes de hemoglobina, prevalência de anemia segundo o trimestre gestacional.

\begin{tabular}{|c|c|c|c|c|c|c|}
\hline \multirow{2}{*}{$\begin{array}{l}\begin{array}{l}\text { Caracteristicas } \\
\text { das gestantes }\end{array} \\
\begin{array}{l}\text { Timestre } \\
\text { gestacional }\end{array}\end{array}$} & \multicolumn{3}{|c|}{ Primigestas } & \multicolumn{3}{|c|}{ Multigestas } \\
\hline & I & II & III & I & II & III \\
\hline $\begin{array}{l}\text { Número de } \\
\text { gestantes }\end{array}$ & 91 & 50 & 10 & 105 & 89 & 18 \\
\hline $\begin{array}{l}\text { Média conc. } \\
\mathrm{Hb}(\mathrm{g} / \mathrm{dl})^{* *}\end{array}$ & $13,40^{*}$ & 12,52 & 12,88 & $13,53^{*}$ & 12,45 & 11,90 \\
\hline $\begin{array}{c}\text { Prevalência de } \\
\text { anemia(\%) }\end{array}$ & $3,3^{* * *}$ & 20,0 & 20,0 & $3,8^{* *}$ & 21,3 & 38,9 \\
\hline
\end{tabular}

- Análise de variância significativa ( $p<0,05)$ : a média da concentraçäo de hemoglobina no primeiro trimestre é significativamente maior que a do segundo e terceiro trimestres, nos grupos das gestantes primigestas e multigestas.

* Teste t-Student não significativo: não houve diferença significativa entre as médias da concentração de hemoglobina das gestantes primigestas, e multigestas em cada trimestre de gestação.

*** Teste do Qui-quadrado entre as prevalências de anemia: a prevalência de anemia no primeiro trimestre é significativamente menor do que as do segundo e terceiro trimestres, no grupo das gestantes primigestas e multigestas.

Das gestantes multigestas $(n=212), 102(48,1 \%)$ tiveram o último parto até há dois anos e em 110 $(51,9 \%)$ gestantes o intervalo do último parto foi há mais que dois anos. Na Tabela 3 săo apresentados as médias da concentração de hemoglobina $e$ prevalência de anemia nas gestantes multigestas com intervalo do último parto até dois anos e intervalo maior que dois anos. Não houve diferença significativa entre as médias das concentraçōes de hemoglobina das gestantes multigestas, com relação ao intervalo do último parto, em cada trimestre de gestação. Não encontramos, também, diferença significativa entre as prevalências de anemia nas gestantes multigestas com intervalo do último parto até dois anos $(17,65 \%)$ e nas multigestas com intervalo do último parto maior que dois anos $(10,91 \%)$.

As prevalências de anemia segundo o número de partos são apresentadas na Tabela 4. Na variável paridade não consideramos os abortos, mas os casos de natimortos.

As gestantes com mais de três partos tiveram prevalência de anemia signifcativamente maior que as com até três partos.

Palgi e col. ${ }^{22}$ citam que a prevalência de anemia aumenta com as gestaçóes subseqüentes como resultado da depleção dos estoques de ferro.

\section{TABELA 3}

Caracterização das gestantes multigestas de acordo con intervalo do último parto(até dois anos de intervalo maior que dois anos), quanto ao número de gestantes, médias das concentraçöes de hemoglobina e prevalência de anemia nos três trimestres de gestação.

\begin{tabular}{|c|c|c|c|c|c|c|}
\hline \multirow{3}{*}{$\begin{array}{l}\text { Caracteristicas } \\
\text { das gestantes } \\
\text { Trimestre } \\
\text { gestacional }\end{array}$} & \multicolumn{6}{|c|}{ Intervalo do último parto } \\
\hline & \multicolumn{3}{|c|}{ Até dois anos } & \multicolumn{3}{|c|}{ Maior que dois anos } \\
\hline & I & II & III & I & II & III \\
\hline $\begin{array}{l}\text { Número de } \\
\text { gestantes }\end{array}$ & 50 & 39 & 13 & 55 & 50 & 5 \\
\hline $\begin{array}{l}\text { Média conc. } \\
\mathrm{Hb}(\mathrm{g} / \mathrm{dl})^{* *}\end{array}$ & $13,45^{*}$ & 12,44 & 11,91 & $13,60^{*}$ & 12,46 & 11,88 \\
\hline $\begin{array}{l}\text { Prevalência de } \\
\text { anemia }\end{array}$ & $6,0^{* * 4}$ & 28,2 & 30,8 & $1,8^{* *+1}$ & 16,0 & 60,0 \\
\hline
\end{tabular}

* Análise de variância significativa $(p<0,05)$ : a média da concentração de hemoglobina no primeiro trimestre é significativamente maior que a do segundo e terceiro trimestres, nos grupos das gestantes com intervalo do último parto até dois anos e intervalo maior que dois anos.

* Teste t-Student não significativo: não houve diferença significativa entre as médias da concentração de hemoglobina das gestantes multigestas com intervalo até dois anos e maior que dois anos em cada trimestre de gestação.

*** Teste do Qui-quadrado entre as prevalências de anemia: significativo $(p<0,05)$, a prevalência de anemia no primeiro trimestre é significativamente menor do que as do segundo e terceiro trimestres, nas gestantes multigestas com intervalo do último parto até dois anos e maior que dois anos.

\section{TABELA 4}

Prevalência de anemia em gestantes da primeira consulta na rede pública, subdistrito do

Butantan, segundo o número de partos das gestantes (paridade).

\begin{tabular}{lcccc}
\hline $\begin{array}{c}\text { Número } \\
\text { de } \\
\text { partos }\end{array}$ & \multicolumn{4}{c}{ Gestantes } \\
\cline { 2 - 5 } & Anêmicas & $\begin{array}{c}\text { Năo } \\
\text { anêmicas }\end{array}$ & Total & $\begin{array}{c}\% \\
\text { Anêmicas }\end{array}$ \\
\hline Nenhum & 15 & 136 & 151 & 9,93 \\
Até três & 21 & 156 & 177 & 11,86 \\
Mais que & 9 & 26 & 35 & $25,71^{*}$ \\
três & & 318 & 363 & 12,40 \\
Total & 45 & 318
\end{tabular}

- Teste do Qui-quadrado entre as prevalências de anemia: significativo $(p<0,05)$, a prevalência de anemia das gestantes com mais de três partos é significativamente maior que as prevalências encontradas nas gestantes com até três partos. 
TABELA 5

Prevalência de anemia em gestantes de primeira consulta segundo o salário mínimo per capita da família (SMPC).

\begin{tabular}{lcccc}
\hline \multirow{2}{*}{ SMPC } & \multicolumn{4}{c}{ Gestantes } \\
\cline { 2 - 5 } & Anêmicas & $\begin{array}{c}\text { Näo } \\
\text { anềmicas }\end{array}$ & Total & $\begin{array}{c}\% \\
\text { Anêmicas }\end{array}$ \\
\hline$<0,5$ & 23 & 79 & 102 & $22,55^{*}$ \\
$0,51-1,0$ & 11 & 123 & 134 & 8,21 \\
$1,01-2,0$ & 8 & 81 & 89 & 8,99 \\
$2,01-3,0$ & 2 & 20 & 22 & 9,09 \\
$>3,0$ & 1 & 10 & 11 & 9,09 \\
Total & 45 & 313 & 358 & 12,57 \\
\hline
\end{tabular}

* Teste do Qui-quadrado entre as prevalências de anemia: significativo $(p<0,05)$, as gestantes que pertenciam a famílias com renda até 0,5 SMPC apresentaram prevalência de anemia significativamente maior que as demais gestantes.

Royston ${ }^{26}$, em sua revisão sobre a prevalência de anemia em mulheres de países em desenvolvimento, notou que vários trabalhos têm sido realizados mostrando que o aumento da paridade é prejudicial à saúde materna no que diz respeito à frequêencia de anemia.

$\mathrm{Na}$ Tabela 5 são apresentadas as prevalências de anemia segundo a renda em SMPC da família da gestante. O SMPC médio foi de 0,98.

A distribuição das gestantes segundo a renda mensal em salário mínimo per capita foi a seguinte: $28,5 \%$ das gestantes pertenciam a familias que tinham renda até $0,5 \mathrm{SMPC} ; 37,4 \%$ tinham renda maior que 0,5 até 1,0 SMPC; $24,9 \%$ tinham renda maior que 1,0 até $2,0 \mathrm{SMPC} ; 6,1 \%$ tinham renda maior que 2,0 até $3,0 \mathrm{SMPC}$ e $3,1 \%$ tinham renda per capita maior que 3,0 SMPC. Alvarenga e col. ${ }^{2}$ estudaram o "índice de status sócioeconômico" da família da mulher grávida que freqüenta o Centro de Saúde Geraldo Paula Souza do Município de São Paulo e encontraram a seguinte distribuição das gestantes, segundo a renda mensal per capita: $29,0 \%$ tinham renda per capita menor que $1,0 \mathrm{SMPC} ; 36,0 \%$ entre 1,0 e menor que $2,0 \mathrm{SMPC} ; 12,0 \%$ entre 2,0 e menor que 3,0 SMPC; $15,0 \%$ entre 3,0 e menor que 4,0 SMPC; $2,0 \%$ entre 4,0 e menor que 5,0 SMPC; $3,0 \%$ entre 5,0 e menor que 6,0 SMPC; e 3,0\% tinham renda superior a 6,0 SMPC.

Encontramos no presente trabalho maior proporção em gestantes com renda mensal per capita até 1,0 SMPC $(65,8 \%)$ quando comparado com o trabalho de Alvarenga e col. ${ }^{2}$. As freqüências das gestantes com mais de um SMPC de renda mensal per capita foram menores do que aquelas encontradas por Alvarenga e col. ${ }^{2}$.

\section{CONCLUSOEES}

Pelo estudo das gestantes de primeira consulta, na rede pública do subdistrito do Butantan, Município de São Paulo, conclui-se que:

- a prevalência de anemia, identificada pela concentração de hemoglobina inferior a $11,6 \mathrm{~g} / \mathrm{dl}$, ocorre em $12,4 \%$ das gestantes estudadas;

- a prevalência de anemia no primeiro trimestre gestacional é significativamente menor que as prevalências no segundo e terceiro trimestres de gestação;

- as gestantes com mais de três partos tiveram prevalência significativamente maior do que as gestantes com até três partos;

- não houve diferença significativa entre as prevalências das gestantes multigestas com intervalo do último parto até dois anos e intervalo maior que dois anos;

- as gestantes que pertenciam a famílias com renda mensal per capita até $0,5 \mathrm{SMPC}$ apresentaram prevalência de anemia significativamente maior.

\section{AGRADECIMENTOS}

Ao Prof. Dr. Paulo E. M. Elias do Departamento de Medicina Preventiva da Faculdade de Medicina da Universidade de São Paulo, pela valiosa colaboração no planejamento e discussão dos aspectos que envolvem a Saúde Pública.

À Diretoria do SUDS R-02, na pessoa da Dra. Maria Cristina Menon, aos Diretores e funcionários dos Centros de Saúde: Escola Butantan, II do Butantan, São Luiz, Rio Pequeno, Vila Borges, Caxingui, Monte Kemel e Real Parque. 
GUERRA, E. M. et al. [The prevalence of anemia in first consultation pregnant women of health centers in a metropolitan area, Brazill. Rev. Saúde públ., S. Paulo, 24: 380-6, 1990.

ABSTRACT: Three hundred and sixty-three pregnant women enrolled in the Pregnancy Medical Care Program of S. Paulo Health Department from the district of Butantan, S. Paulo city, Brazil, were studied at the first routine consultation. At the time they were examined they were not given any medicine containing iron, folic acid or vitamin B12. Their average age was 25 and $65.9 \%$ belonged to families with a monthly per capita income below US\$ 50.00 . Only $3.1 \%$ had a per capita income above U\$\$ 150.00. The prevalence of anemia was $12.4 \%$. The hemoglobin concentration (grams/dl) in the first trimester was significantly higher than in the second and third trimester of pregnancy. The prevalence of anemia in the first trimesters $(3.6 \%)$ was significantly smaller than that found in the second $(20.9 \%)$ and third semesters $(32.1 \%)$. Those women who had had more than three gestations presented a higher prevalence of anemia than those with less than three, and the prevalence of anemia was higher in the group whose families had a monthly per capita income below US\$25.00. analysis.

KEYWORDS: Anemia hypochromic, epidemiology. Pregnancy complications. Hemoglobins,

\section{REFEREANCIAS BIBLIOGRÁFICAS}

1. ALMEIDA, P. A. M. de; CIARI JR., C.; SANTOS, J. L. F.; SIQUEIRA, A. A. F. de. Curva de hemoglobina em um grupo de gestantes normais. Rev. Saúde públ., S. Paulo 7: 273-82, 1973.

2. ALVARENGA, A. T. de; CIARI JR., C.; SANTOS, J. L. F. Indice de status sócio-econômico da família da mulher grávida que freqüenta o Centro de Saúde Geraldo de Paula Souza da Faculdade de Saúde Pública da Universidade de São Paulo. Rev. Saúde públ., S. Paulo, 7: 351-67, 1973.

3. BERQUO, E. S.; SOUZA, J. M. P. de; DAVIDSON, S. L. Bioestatística, São Paulo, Ed. Pedagógica e Universitária, 1981.

4. CARDOZO, L.; ZUNA, H.; URJEL, R.; SAAVEDRA, F. Estudio de las causas de anemia en embarazadas de la maternidad Percy Boland de la ciudade de Santa Cruz - 1985. Bol. Cient. CENETROP, 11: 58-67, 1985 [Separata].

5. COOK, J. D.; ALVARADO, J.; GUTNISKY, A.; JAMRA, M.; LABARDINI, J.; LAYRISSE, M.; LINARES, J.; LORIA, A.; MASPES, V.; RESTREPO, A.; REYNAFARJE, C.; SANCHES-MEDAL, L.; VELEZ, H. ; VITERI, F. A nutritional deficiency and anemia in Latin America: a collaborative study. Blood, 38: 591603,1971

6. COULIBALY, M.; COSTAGLIOLA, D.; ZITIOUN, J.; MARY, J. Y. Modification of hemato-biological parameters in pregnant women in a migrating population in Northern Cameroon; prevalence of anemia, iron and folates deficiencies. Int. J. Vitam. Nutr. Res., 57: 173-8, 1987.

7. DACIE, J. V. \& LEWIS, S. M. Practical haematology. $6^{\text {tb }}$ ed. Edinburgh, Churchill Livingstone, 1984.

8. DEMAEYER, E. \& ADIELS-TEGMAN, M. The prevalence of anaemia in the world. Wld Hlth Statist. Quart., 38: 302-16, 1985.
9. DIEZ-EWALD, M. \& MOLINA, R. A. Iron and folic deficiency during pregnancy in Westem Venezuela. Amer. J. trop. Med. Hyg., 21: 587-91, 1972.

10. FAVElas do Município de São Paulo. Diário Oficial do Municipio, S. Paulo, 2 dez. 1986. p.9.

11. FINCH, C. A. Red cell manual. Seattle, University of Washington, 1969.

12. FLORES, M. E.; RODRIGUES, J.; SANTISTEBAN, I.; ARAUZ, A. G.; CESPEDES, C. Un problema nutricional active la deficiencia de hierro y anemia en la mujer embarazada. Rev. costarric. Cienc. Med., 5 (1): 52-60, 1984.

13. GREBE, G.; LIRA, P.; ARTEAGA, A.; VALENZUELA, M.; CUBILLOS, A. M.; FORADORI, A. Correlacion entre la deficiência de hierro y la ingesta dietetica de hierro en el embarazo. Rev. med. Chile, 107: $989-92,1979$.

14. HO, C. H.; YUAN, C. C.; YEH, S. H. Serun ferritin, folate and cobalamin levels and their correlation with anemia in normal full-term pregnant women. Eur. J. Obstet. Gynec. reprod. Biol., 26: 7-13, 1987.

15. ISAH, H. S.; FLEMING, A. F.; UJAH, J. A. O.; EKWEMPL, C. C. Anaemia and iron status of pregnant and non-pregnant women in the guinea savanna of Nigeria. Ann. trop. Med. Parasit., 79: 485-93, 1985.

16. LAMPARELLI, R. D. V.; BOTHWELL, T. H.; MACPHALL, A. P.; WANDER, W. J.; BAYNES, R. D.; MACFARLANE, B. J. Nutritional anemia in pregnant coloured women in Johannesburg. S. Afr. med. $J ., 73: 477-81,1988$.

17. LIRA, P.; FORADORI, A.; GREBE, G.; LEGUE, M. E.; MUNOZ, B.; ARTEAGA, A.; VELA, P. Caracteristicas hematologicas de una poblacion de embarazadas en Chile. Rev. med. Chile, 106: 343-9, 1978. 
18. LIRA, P.; FORADORI, A.; GREBE, G.; VELA, P. Deficiencia de hierro y folato en mujeres embarazadas de termino. Rev. med. Chile, 112: 127-31, 1984.

19. LORIA, A.; ARROYO, P.; PIEDRAS, J.; MEDAL, L. S. Anemia en el embarazo. II - Datos hematologicos y obstetricos en embarazadas de dos instituciones mexicanas. Rev. Invest. clin., 31: 217-30, 1979.

20. MARTINS, I. S.; ALVARENGA, A. T.; SIQUEIRA, A. A. F.; SZARFARC, S. C.; LIMA, F. D. As determinaçōes biológica e social da doença: um estudo de anemia ferropriva. Rev. Saúde públ., S. Paulo, 21: 73-89, 1987.

21. MAYET, F. G. H. Anaemia of pregnancy. S. Afr. med. J., 67: 804-9, 1985.

22. PALGI, P.; LEVIS,S.; RESHEF, A. Anaemia of pregnancy: evaluation of the effectiveness of routine dietary supplementation program in an Israel community. Amer. J. publ. Hlth, 71: 736-9, 1981.

23. RAZVI, N. Z.; ILAHI, A.; QURESHI, M. J.; HAQ, E.; GILANI, A. H. Problems of the incidence of iron deficiency anaemia in pregnant women under Pakistani conditions. J. Pak. med. Ass., 30 (1): 5-8, 1980.

24. ROMANI, S. A. M.; TORRES, M. A. A.; BATISTA Fo, M. F. M. ; SALZANO, A. C. Anemia em gestantes de duas unidades de saúde da cidade de Recife, Pe. Rev. bras. Malar., 36: 1-10, 1984.

25. RONCADA, M. J. \& SZARFARC, S. C. Hipovitaminose A e anemia ferropriva em gestantes de duas comunidades do vale do Ribeira (Estado de São Paulo, Brasil). Rev. Saúde públ., S. Paulo, 9: 99-106, 1975.

26. ROYSTON, E. The prevalence of nutritional anaemia in women in developing countries: a critical review of available information. Wld Hlth Statist. Quart., 35: 52-91, 1982.

27. SALZANO, A. C.; BATISTA FILHO, M.; FLORES, H.; CALADO, C. L. A. Prevalência de anemia no ciclo gestacional em dois Estados do nordeste brasileiro, Pernambuco e Paraíba. Rev. bras. Pesq. méd. biol., 13: $211-4,1980$.

28. SECRETARIA DE ESTADO DA SAÚDE. Grupo de Trabalho. Implantação do ERSA 2; relatório. São Paulo, 1986. [Mimeografado].

29. SECRETARIA DE ESTADO DA SAÚDE. Grupo de Saúde da Mulher. Pré-Natal Normal. In: Secretaria do Estado da Saúde. Subprograma de Saúde da Mulher. São Paulo, 1988. v.2.

30. SIEGEL, S. Estatistica näo-paramétrica: para as ciências do comportamento. São Paulo, McGraw-Hill do Brasil, 1975.

31. SHUKLA, M. K.; VERMA, B. L.; SARAN, M.; SRIVASTAVA, R. N. A longitudinal study on anaemia of pregnancy in a rural population of Uttar Pradesh. Indian J. med. Res., 75: 541-4, 1982.

32. SZARFARC, S. C. Anemia ferropriva em parturientes e recém-nascidos. Rev. Saúde públ., S. Paulo, 8: 369$74,1974$.

33. SZARFARC, S. C. Comparação entre valores hematológicos (hemoglobina, hematócrito e ferro sérico) da parturiente e do recém-nascido. Rev. Saúde públ., S. Paulo, 9: 43-7, 1975.

34. SZARFARC, S. C.; SIQUEIRA, A. A. F.; MARTINS, I. S.; TANAKA, A. C. D' A. Estudo comparativo de indicadores bioquímicos da concentração de ferro, em duas populaçốes de gestantes, com e sem atendimento pré-natal. Rev. Saúde públ., S. Paulo, 16: 1$16,1982$.

35. SZARFARC, S. C.; SIQUEIRA, A. A. F.; MARTINS, I. S. Avaliação da concentração de ferro orgânico em uma população de grávidas. Rev. Saúde públ., S.Paulo, 17: 200-7, 1983.

36. SZARFARC, S. C. A anemia nutricional entre gestantes atendidas em centros de saúde do Estado de São Paulo (Brasil). Rev. Saúde públ., S.Paulo, 19: 450-7, 1985.

37. VAZ PINTO, A.; PINTO, G.P.; FORMIGA FILHO, J. F. N.; LARA, S.; SANTOS, F. A anemia da gravidez em Sobradinho, cidade satélite de Brasília, Brasil. Rev. bras. Pesq. méd. biol., 8: 381-5, 1975.

38. WORLD HEALTH ORGANIZATION. Scientific Group on Nutritional Anaemias, Geneva, 1967. Report. Geneva, 1968. (Technical Report Series, 405).

39. WORLD HEALTH ORGANIZATION. Expert Committee on the Prevention of Perinatal Mortality and Morbidity, Geneva, 1969. Report. Geneva, 1970. (Technical Report Series, 457).

Recebido para publicação em 27/10/1989 Aprovado para publicaçâo em 17/4/1990 